\title{
Simulation and analysis of HIV-AIDS dynamics
}

\section{Jimbo Henri Claver* and Jesus Pascal}

Department of Applied Mathematics and Statistics, American University of Afghanistan (AUAF), Afghanistan and

Department of Applied Mathematics and Statistics, Waseda University, Tokyo 169-8050, Japan

Email: jimbo.maths@gmail.com

Email:.jpascal@hotmail.com

*Corresponding author

\section{Achile Mbassi}

Department of Urology, Yaounde Central Hospital (YCH), Cameroon and University Hospital, Cameroon

Email:drmbassi@yahoo.fr

\section{Pascal Foumane}

Department Genecology and Obstetric, Yaoundé General Hospital (YGH), Yaoundé, Cameroon Email: pascalfoumane@yahoo.fr

\begin{abstract}
One of the most intriguing questions in mathematical epidemiology are how can one efficiently control and prevent the propagation of a disease. The problem of disease modelling, simulation and control becomes even more fascinating if we look at various risk groups. Referring to HIV-AIDS disease, it is worldwide agreed that the HIV virus seemingly knows when it should attack the body such as to develop AIDS disease. The fundamental question is therefore related to the time and location of such process to happen. To answer to this question, we study a model of propagation of HIV-AIDS in a given population. The AIDS disease is hardly easier to understand than HIV propagation dynamic, but fortunately, we can simplify the system even further by studying the susceptible and infected population dynamics in their behaviour in isolation and/or interaction. Finally, we develop a simulation model based on observed behaviours of susceptible and infected populations. This allows us to test our ideas of how the HIV virus develops into the AIDS disease within the highly controlled environment of computer simulation. Based on these insights, we can suggest new experiments on the actual system and update our models accordingly.
\end{abstract}


Keywords: simulation; disease modelling; management; biodynamic; noise biology; parameter specification; computational biology; biomathematics; HIV-AIDS modelling; stability and computation.

Reference to this paper should be made as follows: Claver, J.H., Pascal, J., Mbassi, A. and Foumane, P. (2019) 'Simulation and analysis of HIV-AIDS dynamics', Int. J. Computational Medicine and Healthcare, Vol. 1, No. 1, pp.16-33.

Biographical notes: Jimbo Henri Claver received his $\mathrm{PhD}$ in Applied Mathematics, Statistics and Computing from the Moscow State University, Russia. He joined the American University of Afghanistan in 2015 as a Professor of Mathematics and Statistics. His research interests include dynamic systems, nonlinear and convex optimisation, quantitative analysis, applied probability, applied statistics, financial mathematics, control theory, risk analysis, medical statistics, modelling and simulation.

Jesus Pascal received his $\mathrm{PhD}$ in Mathematics from the University of Louisiana, USA. He joined the American University of Afghanistan in 2008 as a Professor of Mathematics. His research interests include control theory, quadratic optimisation and operational research.

Achile Mbassi received his $\mathrm{PhD}$ in Medical Science with specialisation in Urology and Surgery from Russia. He is a leading researcher in urology across Africa and active member of medical societies in Africa, Europe and the USA. He has published numerous scientific papers on new developments and frontier research in urology.

Pascal Foumane received his $\mathrm{PhD}$ in Medical Science with specialisation in Genecology and Obstetric. He is a Professor at the CHU-Yde and Physician at the HGY-Yde, Cameroon. He has conducted various research in genecology and obstetric. He is author of many publications in scientific reviews, journals and book chapters. He is a leading research in medical research in Cameroon and internationally.

\section{Introduction}

Worldwide, it is estimated that one million people died from AIDS every year and another 30 million live with the disease (WHO, 1990). The cause of the HIV-AIDS disease seems now well established and its prevention well understood. Scientists from various research backgrounds have been working hard to improve the understanding of the disease progression; however, mathematical modelling and statistical approaches have been only lately introduced in this research in order to better understand the disease dynamic in a quantitative manner and predict important features such as appropriate drug dosage, the thresholds of various parameters that affect the progression of the disease and many others (Anderson et al., 1986; Anderson and May, 1991; Anderson and Garnett, 2002). It should be underlined that dynamic models and computer simulations are experimental tools for comparing regions or risk groups, testing theories, assessing quantitative conjectures and answering relevant questions. Basically, there are three approaches to the modelling of HIV-AIDS. The first and more direct approach is to 
predicting AIDS in the future by extrapolation (Morgan and Curran, 1989; Carr, 1981; Butler and Waltman, 1986). This method fits the hypothetical form of the AIDS incidence data in recent years and then extends this curve by several years as a prediction of AIDS cases in the future. This method assumes that the current trends will continue for at least a few years in the future. Often separate curves and extrapolations are done for various risk groups. Some advantages of extrapolation are its simplicity and ease of use. The extrapolation method is a good predictor of AIDS incidences for a few years into the future, but it is not good for longer future forecasts because it does not consider changes in the HIV epidemic group due to factors such as behavioural changes or saturation in the high risk group, and also it does not give information on HIV incidence or mechanisms (Bollinger et al., 1997). The second approach is based on back calculation (Butler and Waltman, 1986; Gibbs and Tess, 1999). The total number of AIDS cases at time $t$ is the summation up to time $t$ of the product of the HIV incidence at time $\tau$ and the probability of developing AIDS within $t-\tau$ years after infection. Thus, if the HIV incidence and the distribution of the AIDS incubation period were known up to time $t$, then the cumulative number of AIDS cases may be calculated in a straightforward manner. Back calculation is a deconvolution process - it uses a given AIDS incidence up to time $t$ and an estimated distribution for the AIDS incubation period to estimate the HIV incidence up to time $t$ (Morgan and Curran, 1989). This incidence up to time $t$ and its extrapolation for several years are then used to forecast the AIDS incidence for a period of several years.

The distribution of the incubation period for AIDS can be estimated parametrically or non-parametrically (Aggarwal et al., 1997). This method has the advantage that it also yields estimates of HIV incidence. However, there are several disadvantages: it does not yield information on the HIV transmission dynamic or estimates of parameter values. Estimated distributions for the AIDS incubation period are uncertain and the back calculation procedure is very sensitive to the distribution used (Diekmann and Metz, 1990). This instability of the back calculation process implies that the confidence intervals of estimates of HIV incidence and future AIDS incidence are very wide. Finally, the third approach to modelling AIDS is to use HIV transmission dynamic models which include the progression to AIDS. These models often have populations divided into compartments consisting of those who are susceptible in each of the infection stages, or in the AIDS phase. In deterministic transmission models, the movement between these compartments by becoming infected, progressing to the next stage or AIDS migrating or dying is specified by system of difference or differential equations (Smith, 1988). Modelling can also be used to theoretically evaluate, compare or optimise various detection, prevention, intervention and control programs. HIV-AIDS transmission dynamic models are stochastic with various probabilities of moving to the next stage at each time step. Morgan and Curran (1989) investigated the relationship between Monte Carlo simulations of stochastic HIV-AIDS models and solution of deterministic model using the expected values. Further, Nowak and May (1991) looked at a general stochastic model of the simulation of HIV transmission and AIDS. However, these models suffer from lack of flexibility and adaptability. Also until now almost all proposed models being used are of deterministic form, and are sometimes speculated to be approximations of stochastic models (Gibb and Tess, 1999; Mukandavire et al., 2007). Explicit stochastic models have not yet been used. In this work, we propose a new model which takes into consideration the sensitivity of the disease propagation and time delay in both the susceptible and infected subpopulations. Our model is an integrative version of the system of stochastic delayed differential equation SSDDEs introduced earlier in 
(Jimbo and Craven, 2011; Jimbo and Mathew, 2012) for modelling complex dynamical systems. Our paper is organised as follows: In Section 2, we introduce the novel model. In Section 3, we present the simulation results, we also show the superiority of this model over the existing models. In Section 4, we develop and present the local stability of disease free equilibrium. We finally end this work in Section 5 with a short conclusion.

\section{Modelling framework}

The hypothetical model of HIV-AIDS divides the population into sub-populations of susceptible individuals (S), HIV infected with no clinical symptoms of AIDS (I) and HIV infected individual with AIDS symptoms (A). We assume that the susceptible individuals are recruited into the population at per capital rate $\Lambda$ and susceptible individual acquire HIV infection following contact with HIV-infected individual at a rate $\lambda$. Without loss of generality and spending too much time on biological aspect of the problem, we turn to the mathematical interpretation of biodynamic and derive the equations of the epidemiological model which is the extension of previous models that have been recently proposed.

\subsection{Previous model}

\subsubsection{Deterministic epidemiological model}

The model dynamic considers the chart flow (Figure 1) where S, I and A represent respectively the suspected, infected without and with symptoms of AIDS individuals. The variables $b$ represents the birth rate, $k$ the transmission rate from susceptible to infected and $r$ the recovery rate $d_{0}$ the death rate that can increase or decrease depending on the population. We assume that the time course of the disease in a given population is short compared to the lifespan of the individuals, so that there is no self-reconstitution.

Figure 1 Schematic representation of disease dynamic

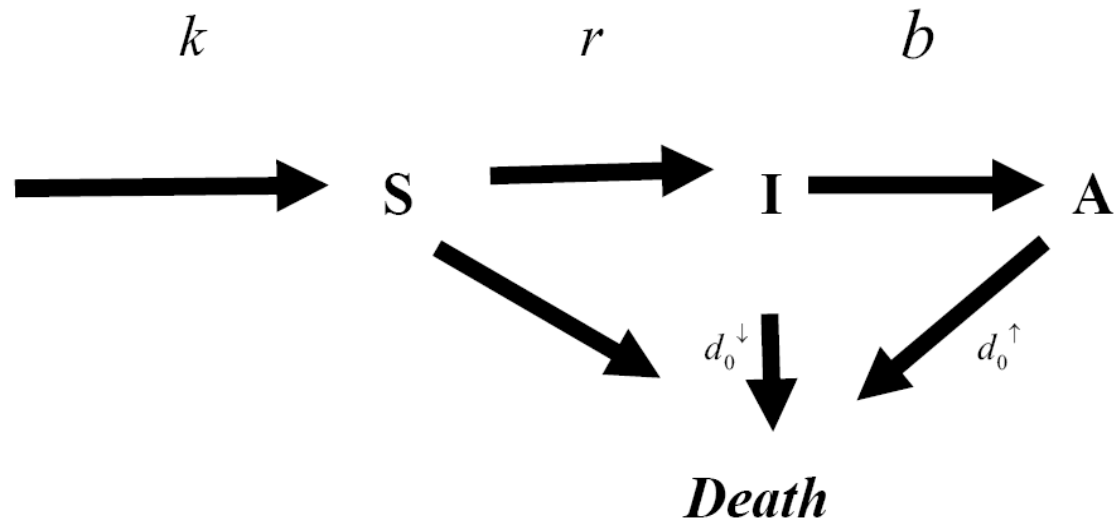

At a given point in time, part of a population $P$ is infected at a rate $\alpha$ and among the infected population a proportion will develop the disease at a rate $\beta$. Following the 
schematic representation of disease dynamic in Figure 1, we derive the system of equations:

$$
\left\{\begin{array}{l}
\frac{d S}{d t}=b S-k I S \\
\frac{d I}{d t}=k I S-r I
\end{array}\right.
$$

As a first stage simplification, we put $\frac{d A(t)}{d t}=0$ where $S$ and $I$ represent respectively the suspected and infected individuals (populations) in the non-fatal disease consideration.

Figure 2 Compartmental representation of disease dynamic (see online version for colours)

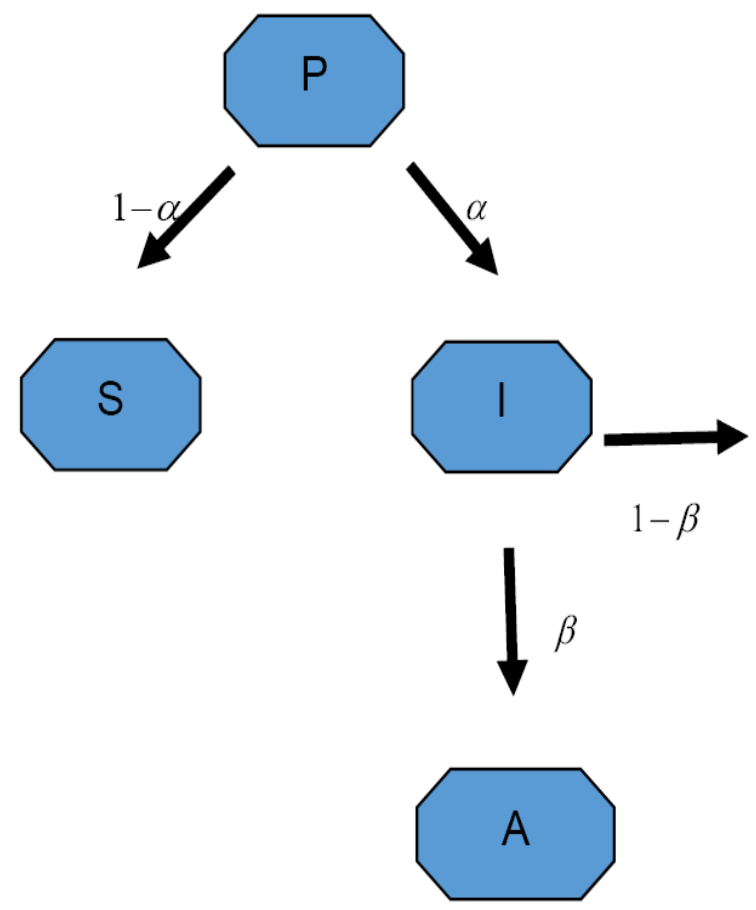

\subsection{Novel model}

\subsubsection{Stochastic epidemiological model}

The stochastic model of HIV-AIDS disease progression is relatively new. Although the field of epidemiology has a long well established history, it is only within the past several decades that mathematicians and epidemiologists have begun to work together to create model that attempts to predict the progression of disease in given population. Here, we propose a new model to analyse the dynamic of the disease and its progression. Our model is based on a system of delay stochastic differential equations (DSDEs) used to capture various scenarios of evolution of disease in a chosen population. We believe that 
introducing independent time delays in susceptible and incident dynamic will not only improve the model by capturing more interesting features but also support idea of nonlinear progression of disease that has already been observed in several patient at hospitals (Brauer and Castillo-Chavez, 2001; Jimbo and Craven, 2011). We now state the continuous time stochastic differential equation for the model. This is a system of nonlinear initial value stochastic differential delay equations (SDDEs). We assume that the probability distribution noise in susceptible and infected dynamics is of different nature. In susceptible dynamic, the noise is normally distributed with men 0 and variance $\sigma^{2}$ rather in incident dynamic the noise is Poisson distributed with mean $\lambda=2$.

$$
\left\{\begin{array}{l}
\frac{d S(t)}{d t}=(b-k I) S\left(t-\tau_{S}\right)+\xi_{S}(t) \\
\frac{d S(t)}{d t}=(k S-r) I\left(t-\tau_{I}\right)+\xi_{I}(t)
\end{array}\right.
$$

where $\xi_{S}(t) \sim N\left(0, \sigma^{2}\right) ; \xi_{I}(\mathrm{t}) \sim \mathrm{P}_{0}(2)$ represent the sources of extrinsic noise, $\tau_{S}, \tau_{I}$ are respectively the Susceptible and Infected individual delays and contribute to the intrinsic noise source of noise. There is no possible correlation between $\xi_{S}(t)$ and $\xi_{I}(t)$ noise dynamics. For this reason, we set $\operatorname{corr}\left(\xi_{S}(t), \xi_{I}(t)\right)=0$. Next, we perform the simulation of the model and present some new results.

\section{Simulation and result}

Using MATLAB software, we produce the solutions that follow.

\subsection{Setup}

The simulation parameters are set according biophysical, biodynamical and clinical considerations (Diekmann and Metz, 1990; Nowak and May, 1991; Nicolosi and Musicco, 1994). It is generally agreed that biochemical, biophysical and biodynamical parameters often depend on the state of the patients and or drug consumption. In healthy individual, these parameters are stable, but in unhealthy patients, they tend to me more unstable or noisier (Van Den Driessche and Watmough, 2002). Relaying on the above assumptions and the data that have being used in Castillo-Chavez et al. (2002) lead us to the following parameter specifications.

\section{Specification of the parameters of the model}

$$
\begin{aligned}
& b=0.001 ; \quad k=0.04 ; \quad r=0.02 ; \quad t_{s}=0.001 \\
& S_{0}=100 ; \quad \tau_{s}=1 ; \quad \tau_{I}=3 ; \quad I_{0}=10
\end{aligned}
$$

\subsection{Results}

The results present the results of the simulation. 
Figure 3 Summarisation of the effect of the parameters on the distribution of the incidence population over generation periods (see online version for colours)

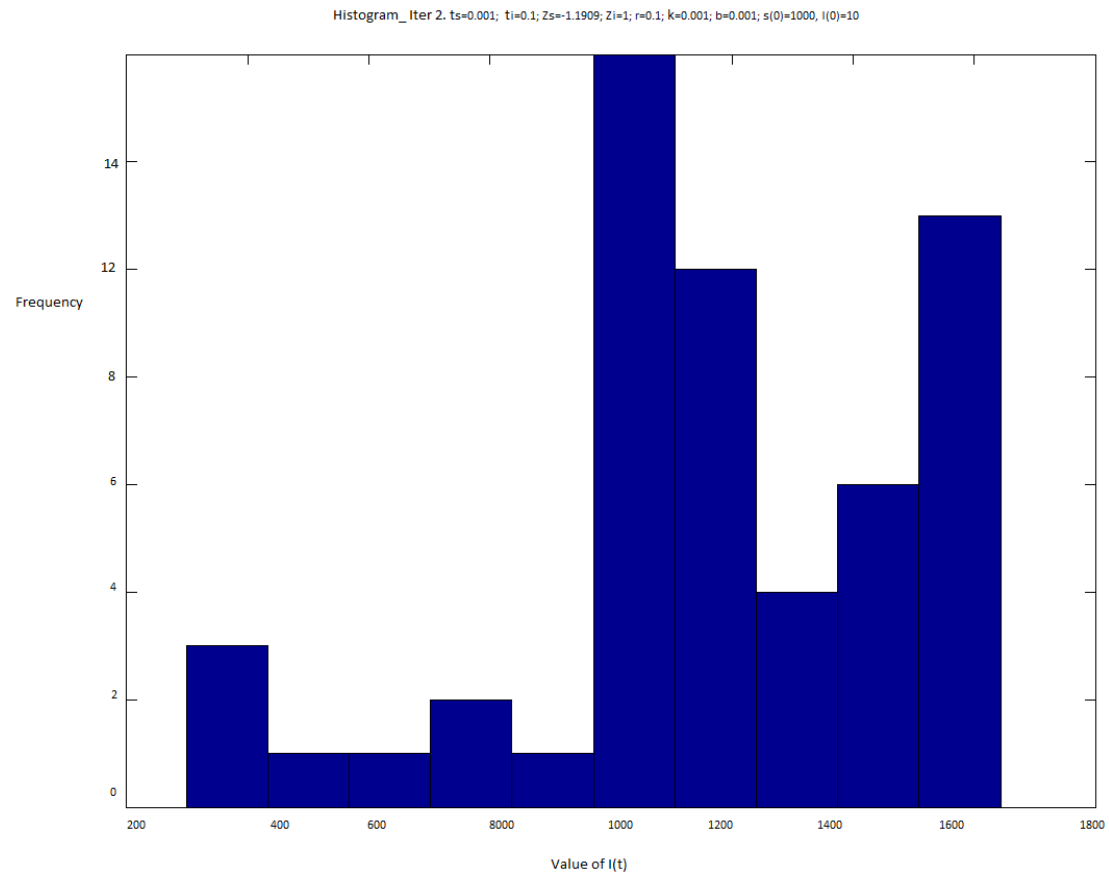

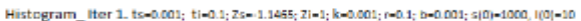

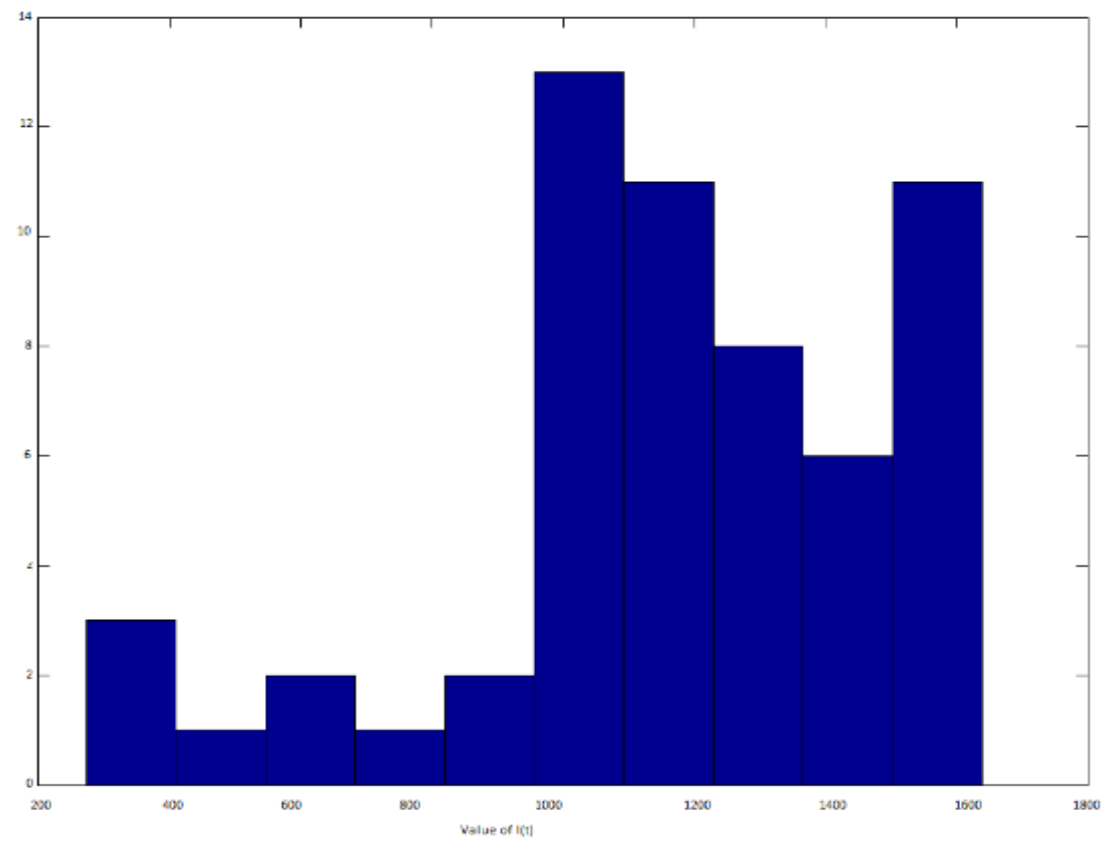

Note: It is clear that over generation time the shape of the distribution tends to become normalised. 
Figure 3 Summarisation of the effect of the parameters on the distribution of the incidence population over generation periods (continued) (see online version for colours)
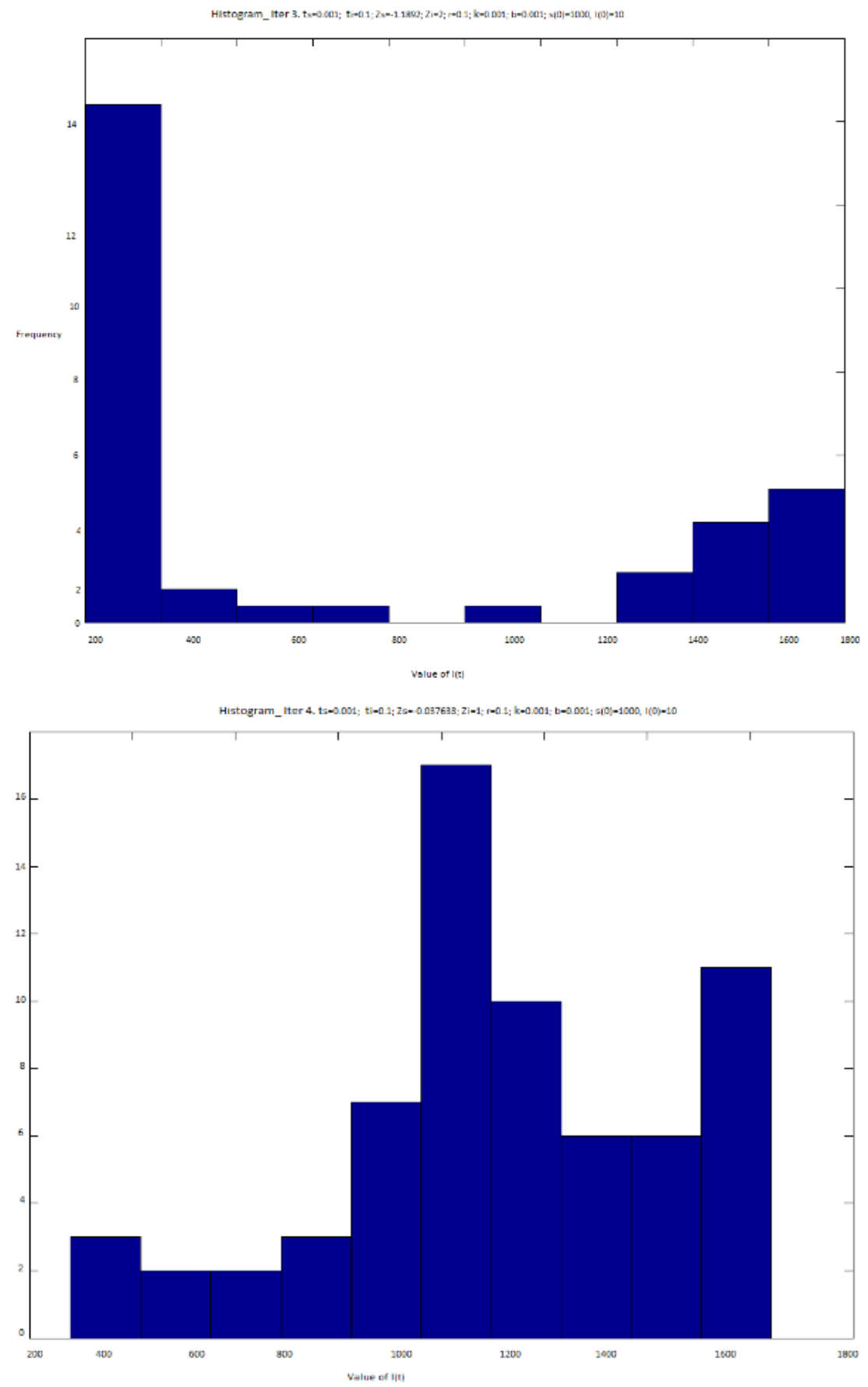

Note: It is clear that over generation time the shape of the distribution tends to become normalised. 
Figure 4 Solutions for $S(t), I(t)$ (see online version for colours)
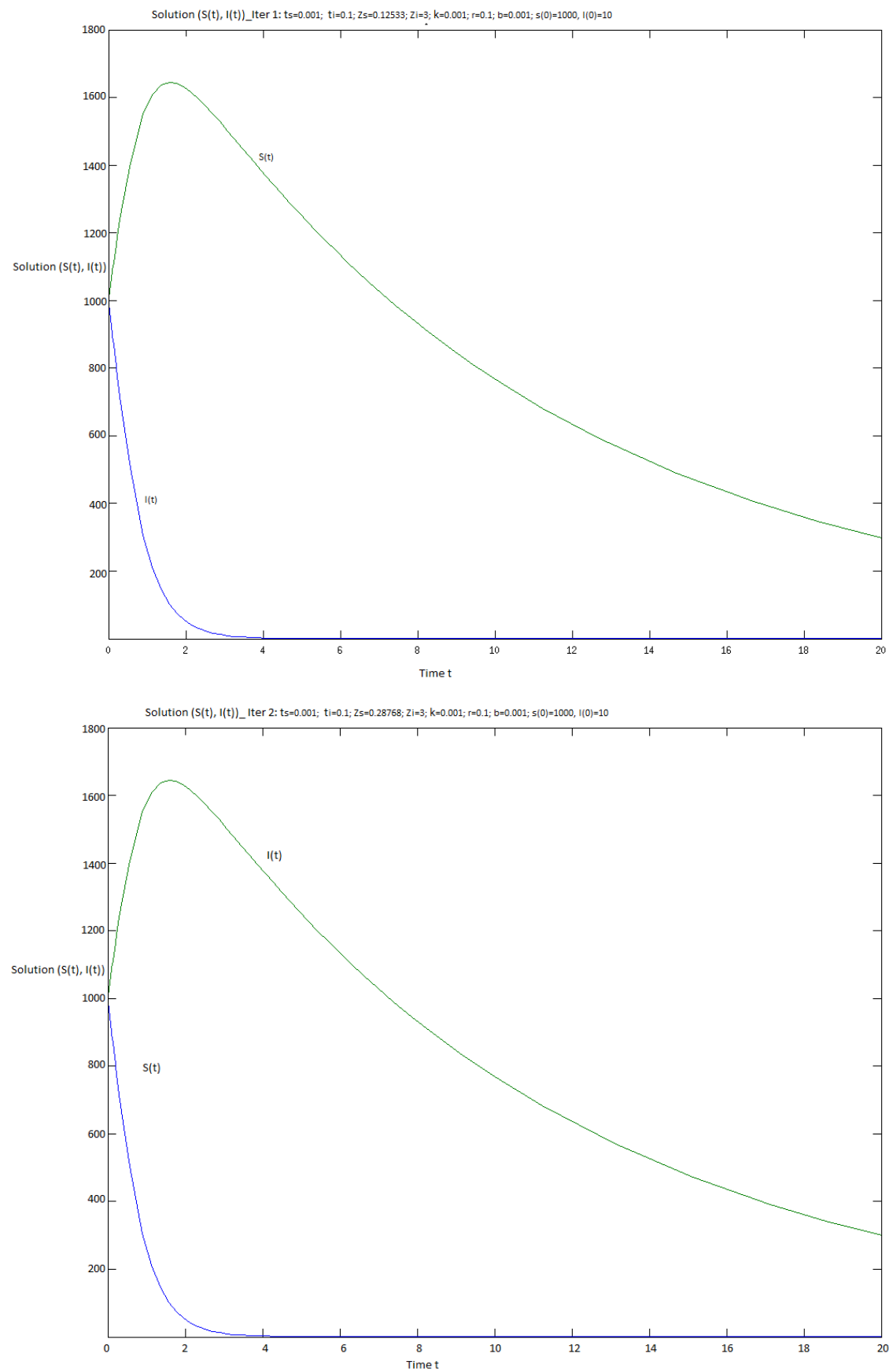

Notes: It can be seen that over time, while $I(t)$ reaches its maximum, $S(t)$ reaches it minimum at the same time indicating that as the number of incident population will decrease the number of susceptible population will increase. 
Figure 4 Solutions for $S(t), I(t)$ (continued) (see online version for colours)
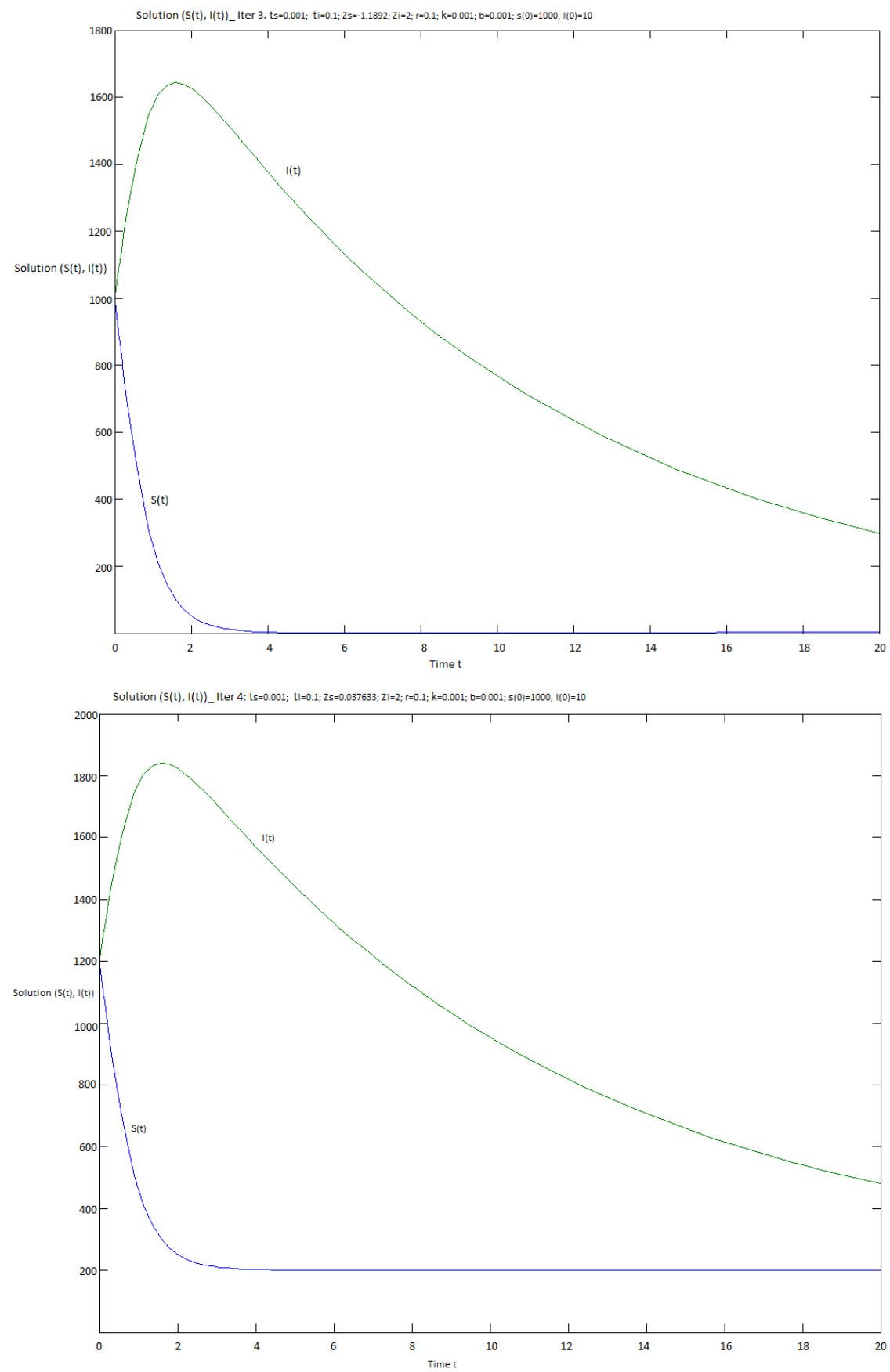

Notes: It can be seen that over time, while $I(t)$ reaches its maximum, $S(t)$ reaches it minimum at the same time indicating that as the number of incident population will decrease the number of susceptible population will increase. 
Figure 5 Scatter plot of $I(t)$ population (see online version for colours)
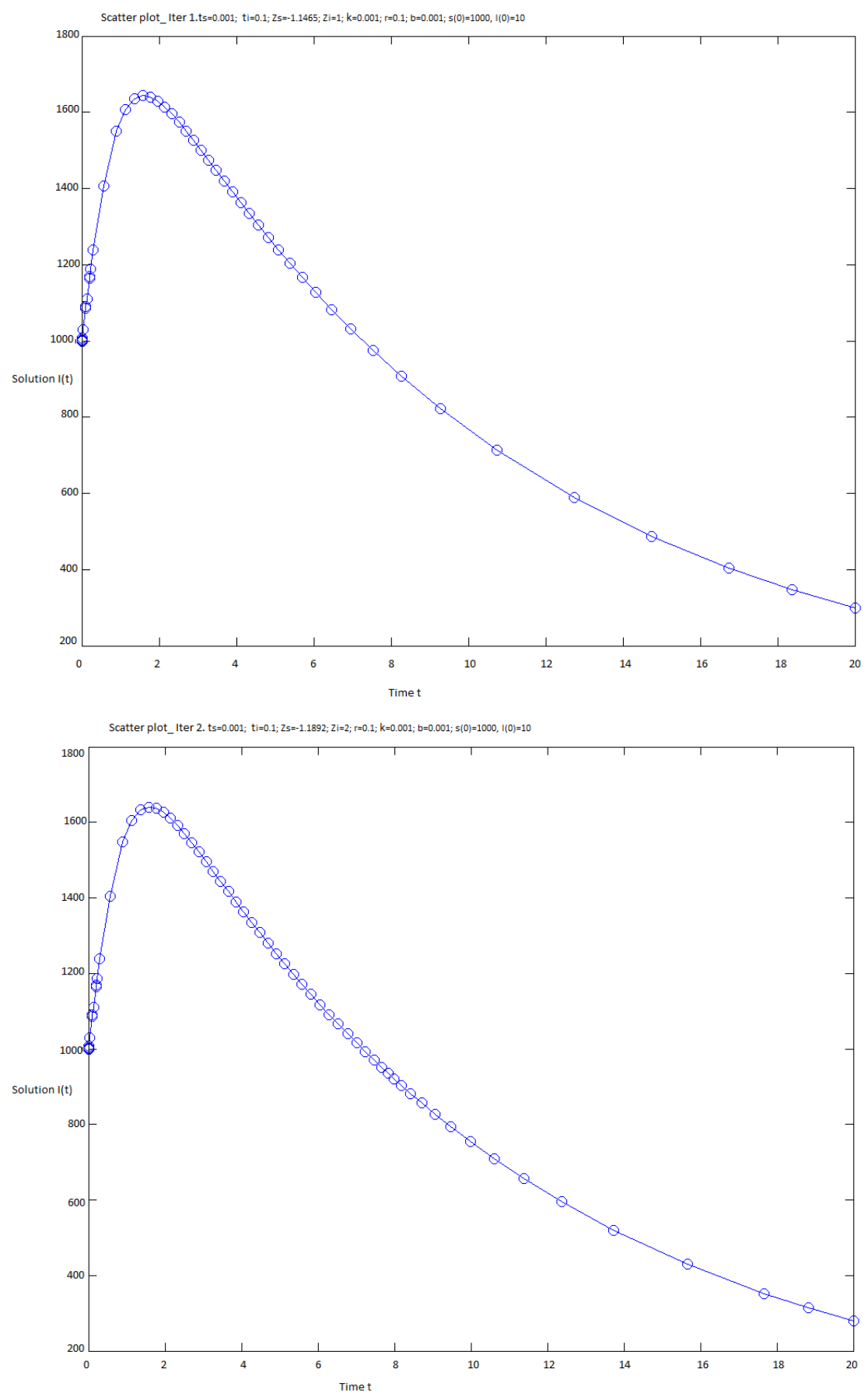

Notes: We observe an acceleration of the number of incident population around the peak level and considerable reduction of this number as we move further to the maximum, this shows the positive effect of learning over time. 
Figure 5 Scatter plot of $I(t)$ population (continued) (see online version for colours)
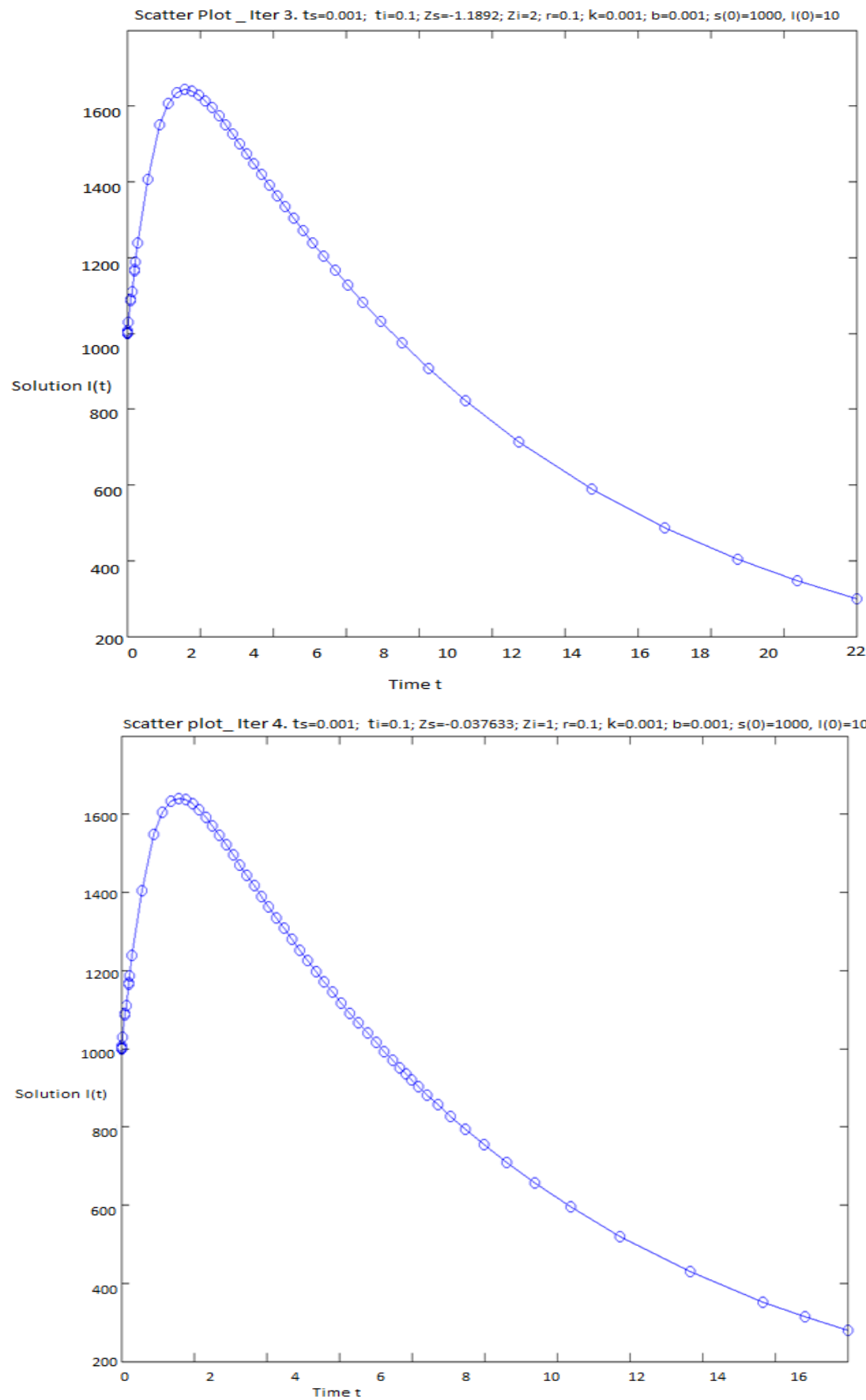

Notes: We observe an acceleration of the number of incident population around the peak level and considerable reduction of this number as we move further to the maximum, this shows the positive effect of learning over time. 
Figure 6 Solution $S(t)$ (see online version for colours)
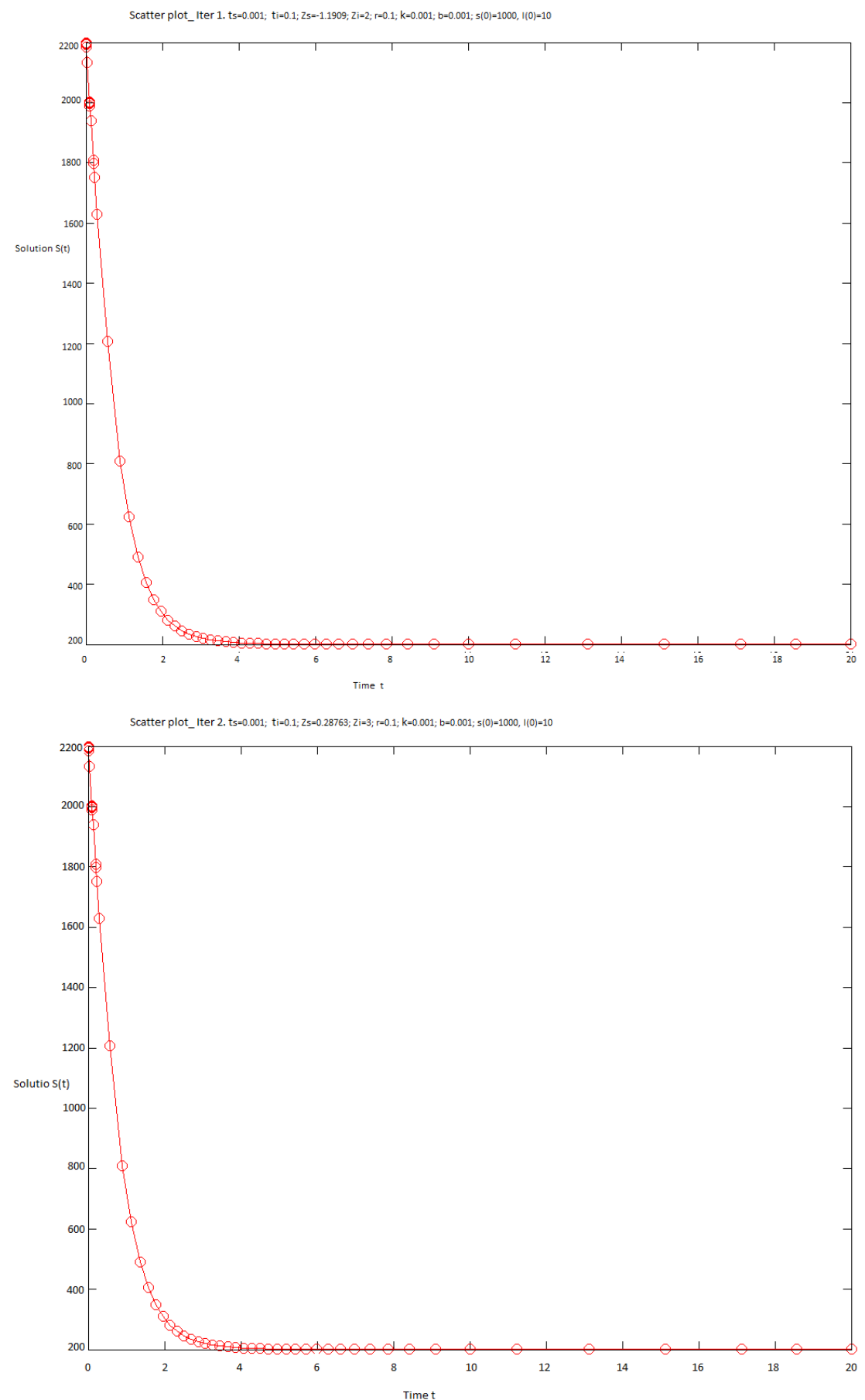

Notes: It can be seen that over time, the number of susceptible population reduces considerably indicating that as people know more about the risk exposure they tend to be more cautious. 
Figure 6 Solution $S(t)$ (continued) (see online version for colours)
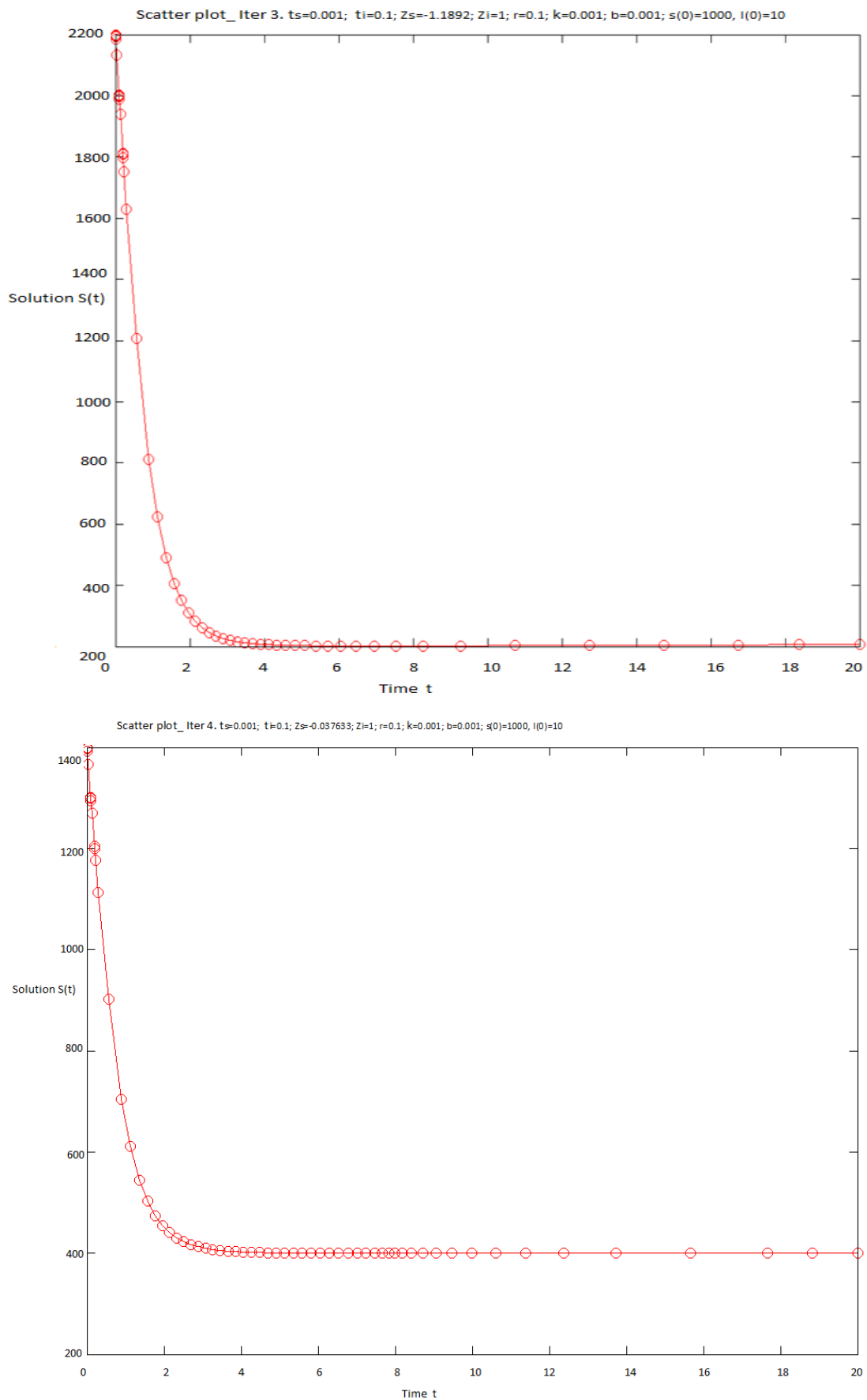

Notes: It can be seen that over time, the number of susceptible population reduces considerably indicating that as people know more about the risk exposure they tend to be more cautious. 


\section{Local stability and disease - free equilibrium}

Let us assume the total interacting sub-populations equals to $N(t)=S(t)+I(t)+A(t)$. It is known that natural death occurs in all human sub-population at rate $\mu$ and recruitment rate $\Lambda$. Further, there is a HIV-AIDS constant $\delta$ in AIDS class. We extend model (2) and recall the following:

$$
\left\{\begin{array}{l}
S^{\prime}(t)=\Lambda-\frac{(b-k I)}{N}\left(t-\tau_{S}\right)+\xi_{S}(t) \\
I^{\prime}(t)=\frac{(I+\eta A)}{N}-(\mu-k) I\left(t-\tau_{I}\right)+\xi_{I}(t)
\end{array}\right.
$$

Assumption 1: At equilibrium $\left\{\begin{array}{l}t-\tau_{S}=t-\tau_{I}=\Delta t=1 \\ \xi_{S}(t) \triangleq \xi_{I}(t) \mapsto \varepsilon\end{array}\right.$.

Applying Assumption 1, equation (3) becomes

$$
\left\{\begin{array}{l}
S^{\prime}(t)=\Lambda-\frac{(b-k I) S}{N} \\
I^{\prime}(t)=\frac{(I+\eta A)}{N}-(\mu-k) I
\end{array}\right.
$$

with $A^{\prime}=k I-(\mu+\delta) A$.

Assumption 2: Consider the region $\phi=\left\{(S, I, A) \in R_{1}^{3}: N \leq \Lambda / \mu\right\}$. Any solution of model (4) starting in $\phi$ remain in $\phi$ for all $t \geq 0$, thus $\phi$ is positively invariant.

The HIV-AIDS model (4) has a disease - free equilibrium given by $\varepsilon_{0}=\left(\frac{\Lambda}{\mu}, 0,0\right)$; it is obvious that $\varepsilon_{0}$ attract the region stable manifold $\varepsilon_{0}$ :

$$
\phi_{0}=\{(S, I, A) \in \phi: I=A=0\}
$$

The linear stability of $R_{0}$ is governed by the basic reproductive number $R_{0}$. The stability of this equilibrium will be investigated using the next generation operator (Smith, 1988; Carr, 1981; Jimbo and Craven, 2011). Using the notation in Mehendale et al. (1996) and Mukandavire et al. (2007) on model system (2), the matrices $F$ and $V$ for the new infection terms and the remaining transfer terms are respectively given by:

$$
F=\left(\begin{array}{cc}
\beta & \beta \eta \\
0 & 0
\end{array}\right) \text { and } V=\left(\begin{array}{cc}
\mu+k \beta & 0 \\
-k & \mu+\delta
\end{array}\right)
$$

As in Assumption 1, it follows that the basic reproductive number, denoted by $R_{0}$ and given by:

$$
R_{0}=\rho\left(F V^{-1}\right)=\frac{\beta(\delta+k \eta+\mu)}{(k+\mu)(\delta+\mu)}
$$


Lemma 1: The disease-free equilibrium of HIV-AIDS model of system of equation (4) is locally asymptotically stable if $R_{0}<1$ and unstable if $R_{0}>1$.

The basic reproductive number measures the average number of new infections generated by a single infected individual in a completed susceptible population. Thus, Lemma 1 implies that HIV-AIDS can eliminated from the community (when $R_{0}<1$ ) if the initial sizes of the sub-populations of the model are in the basin of attraction of the disease -free equilibrium $\varepsilon_{0}$.

Theorem 1: Whenever $R_{0}<1$, the disease-free equilibrium of the simplified model (4) is given by $\varepsilon_{0}$ which is globally stable in $\phi$ and unstable in $R_{0}>1$.

Proof: We consider the following Liapunov function $\ell: \ell=(\mu+\delta+\mu \eta) I(t)+\eta(\mu+\kappa) A(t)$, its derivative along the solution to model (4) is $\ell^{\prime}(\mu+\delta+\mu \eta) I^{\prime}(t)+\eta(\mu+\kappa) A^{\prime}(t)$.

$$
\begin{aligned}
\ell^{\prime} & =(\mu+\delta+\mu \eta)\left(\beta(I+\eta A) \frac{S}{N}-(\mu+\kappa) I\right)+\eta(\mu+\kappa)(\kappa I-(\mu+\delta) A) \\
& =(\mu+\delta+\mu \eta)\left(\beta(\mu+\delta+\eta \kappa) \frac{S}{N}-(\mu+\delta)(\mu+\kappa) I\right) \\
& +\eta\left(\beta(\mu+\delta+\eta \kappa) \frac{S}{N}-(\mu+\delta)(\mu+\kappa) A\right) \\
& =\left(\beta(\mu+\delta+\eta \kappa) \frac{S}{N}-(\mu+\delta)(\mu+\kappa) I\right)+\eta\left(\beta(\mu+\delta+\eta \kappa) \frac{S}{N}-(\mu+\delta)(\mu+\kappa) A\right)
\end{aligned}
$$

We apply the approximation for $S \leq N \leq(\beta(\mu+\delta+\eta \kappa)-(\mu+\delta)(\mu+\kappa) I)+\eta(\beta(\mu+\delta+$ $\eta \kappa)-(\mu+\delta)(\mu+\kappa) A)=(\mu+\delta)(\mu+\kappa)\left(R_{0}-1\right) I+\eta(\mu+\delta)(\mu+\kappa)\left(R_{0}-1\right) A \leq 0$ for $R_{0}<1$. Assuming that all the model parameters and non-negative, it follows that $\ell$ for $R_{0}<1$ with $\ell$ if and only if $I=A=0$. Thus, $\ell$ is a Liapunov function on $\phi$. Since $\phi$ is invariant and attracting, we have that the largest compact invariant set in $\{(S, I, A) \in \varphi: \ell=0$ is the singleton $\left\{\varepsilon_{0}\right\}$, and by the LaSalles's invariant principle (LaSalle, 1976), any solution of equation (4) with initial condition in $\phi$ approaches $\varepsilon_{0}$ as $t \rightarrow+\infty$. Finally, we have obtained that $(S(t), I(t), A(t)) \rightarrow(\Lambda / \mu, 0,0)$ and $\varepsilon_{0}$ is globally asymptotically stable in $\phi$ as long as $R_{0}<1$.

\section{Conclusions}

The study of HIV-AIDS dynamical model is proposed in this work. The Liapunov function and LaSalle's invariant principle are used to provide global stability of the equilibrium of the model. We show that the model is positively invariant. Further, the basic reproduction number expression is derived using the next generation matrix method. We also do some analysis on the stability of the steady points of the model. Finally our result shows that the disease can be well controlled when $R_{0}<1$. The advantage of this approach is that it is not reliant much on parameters. Its disadvantage, however, is that it is largely dependent on assumptions that might not be verifiable. 


\section{Acknowledgements}

We are grateful to all colleagues for discussions and suggestions which helped us to improve the presentation of this paper. We are equally grateful to Mr. Hamidullah Hamidy for reviewing the final version of this paper.

\section{References}

Aggarwal, O.P., Sharma, A.K. and Indrayan, A. (1997) HIV/AIDS Research in India, National AIDS Control Organization, New Delhi.

Anderson, R. and May, R. (1991) Infectious Diseases of Humans, Oxford University Press, London/New York.

Anderson, R.M. and Garnett, G.P. (2002) 'Mathematical models of the transmission and control of sexually transmitted diseases', Sex Transm. Dis., Vol. 27, No. 10, pp.636-643.

Anderson, R.M., Medley, G.F., May, R.M. and Johnson, A.M. (1986) 'A preliminary study of the transmission dynamics of the human immunodeficiency virus (HIV), the causative agent of AIDS', IMA J. Math Appl. Med. Biol., Vol. 3, No. 4, pp.229-63.

Bollinger, R.C., Brookmeyer, R.S. and Mehendale, S.M. (1997) 'Risk factor and clinical presentation of acute primary HIV infection in India', JAMA, Vol. 278, No. 23, pp.2085-2089, DOI: $10.1001 /$ jama.1997.03550230061038.

Brauer, F. and Castillo-Chavez, C. (2001) 'Mathematical models in population biology and epidemiology', in 221 Texts in Applied Mathematics Series, Vol. 40, Springer-Verlag, New York.

Butler, G. and Waltman, P. (1986) 'Persistence in dynamical systems', in Proceedings of the American Mathematical Society, Vol. 96, No. 223, pp.425-430.

Carr, J. (1981) Applications of Centre Manifold Theory, Springer-Verlag, New York.

Castillo-Chavez C., Feng Z. and Huang W. (2002) 'On the computation of RO and its role in global stability', Institute for Mathematics and its Applications, Vol. 125, p.229.

Diekmann, O. and Metz, J.H. (1990) 'On the definition and computation of the basic 230 reproduction ratio $\mathrm{r} 0$ in models for infectious diseases in heterogeneous populations', Journal of Mathematical Biology, Vol. 231, pp.365-382.

Gibb, D.M. and Tess, B.H. (1999) 'Interventions to reduce mother-to-child transmission of HIV infection: new developments and current controversies', AIDS, Vol. 1, No. 3, Suppl. A, pp.S93-102.

Jimbo, H.C. and Craven, M.J. (2011) 'Unconstrained optimization in a stochastic cellular automata system', Journal of Nonlinear Analysis and Optimization, Vol. 1, No. 2, pp.103-110.

Jimbo, H.C. and Mathew, C.J. (2012) 'Modelling, simulation and analysis of noise in biological systems', Journal of Control and Application, No. 80, pp.23-35, IASTED, DOI: 102316/ P2012.781-03/.

LaSalle (1976) The Stability of Dynamical Systems, CBMS-NSF Conference Series in Applied Mathematics, Vol. 25, SIAM, Philadelphia, USA.

Mehendale, S.M., Shepherd, M.E. and Divekàr, A.D. (1996) 'Evidence for high prevalence and rapid transmission of HIV among individuals attending STD clinics in Pune, India', Indian J. Med Res., Vol. 104, pp.317-335.

Morgan, J.I. and Curran, T. (1989) 'Stimulus-transcription coupling in neurons: role of cellular immediate early gene', Trends Neurosci., November, Vol. 12, No. 11, pp.459-462.

Mukandavire, Z., Garira, W. and Chiyaka C. (2007) 'Asymptotic properties of an HIV/aids model with a time delay', Journal of Mathematical Analysis and Applications, Vol. 330, No. 2, pp.916-933. 
Nicolosi, A. and Musicco, M. (1994) 'Risk factors for woman-to-man sexual transmission of the human immunodeficiency virus', Journal of Acquired Immune Deficiency Syndromes, Vol. 7, pp.296-300.

Nowak, M.A. and May, R.M. (1991) 'Mathematical biology of HIV infections: antigenic variation and diversity threshold', Math Biosci., Vol. 106, No. 1, pp.1-21.

Smith, H. (1988) 'Systems of ordinary differential equations which generate an order preserving flow', SIAM Review, Vol. 30, No. 1, pp.87-113.

Van Den Driessche, P. and Watmough, J. (2002) 'Reproduction numbers and sub-threshold endemic equilibria for compartmental models of disease transmission', Mathematical Biosciences, pp.29-48.

WHO (1990) World Health Organization Review. 